\title{
Production de transferrine par les oligodendrocytes dans le cerveau adulte
}

\author{
Bertrand Bloch, Theodora Popovici, \\ Mariano J. Levin, David Tuil, Axel Kahn
}

\section{Summary}

Presence and production of transferrin in the adult rat brain have been investigated, using combined immunohistochemistry and in situ hybridization technique on tissue sections. Indirect immunofluorescence with five distinct antibodies against rat and human transferrin (including a monoclonal antibody) demonstrated staining of the cytoplasm of oligodendrocytes (a category of glial cells) in most parts of the brain, especially in the white matter. In situ hybridization procedure using as a probe rat transferrin complementary DNA labeled with ${ }^{32} \mathrm{P}$ demonstrated the presence of transferrin mRNA in glial cells whose aspect, topography and organization exactly matched the ones of cells stained with transferrin antisera. These results provide evidence that the transferrin gene is expressed in the central nervous system and that transferrin is produced and stored by oligodendrocytes in the adult rat brain. These data suggest that this molecule could have specific functions in relation to the nervous system activity.

\section{ADRESSES}

B. Bloch : Laboratoire d'histologie, Faculté de Médecine, 4. place Saint-Jacques, 25030 Besançon cedex.

T. Popovici : Unité Inserm U I98, route de Dôle, 25000 Besançon.

M.-J. Levin, D. Tuil, A. Kahn : Laboratoire de génétique et de pathologie moléculaires, Unité Inserm I 29, 24, rue du Faubourg-Saint-Jacques,
- étude de la transferrine suscite depuis quelques années un regain d'intérêt en raison de certaines propriétés nouvellement mises en évidence de cette molécule. Outre son rôle dans le transport sérique du fer [1] et dans sa cession aux cellules de la lignée érythropoḯtique [1], la transferrine apparaît comme un facteur indispensable à la prolifération, à la différenciation et à la maturation de nombreux types cellulaires in vitro [2] et probablement in vivo [3]. En particulier, la transferrine a été identifiée comme un facteur endogène capable de promouvoir la différenciation du muscle et du rein au cours du développement $[3,4]$.

Divers résultats montrent, qu'outre le foie, il existe d'autres sites de synthèse de transferrine dans l'organisme, tels l'oviducte [5] et le testicule [6]. Par ailleurs, il a été mis en évidence récemment une présence d'ARN messager (ARNm) de la transferrine dans le muscle strié [7] au cours du développement et dans le système nerveux central, chez le poulet [5] et le rat [7]. Chez cette dernière espèce, la quantité d'ARNm présente augmente progressivement à partir de la naissance pour atteindre un plateau à l'âge adulte [7]. L'expression du gène de la transferrine dans le cerveau du rat adulte est indépendante des facteurs qui la font varier au niveau du foie, en particulier la charge en fer de l'organisme [8].

Ces observations nous ont conduit, dans le présent travail, à rechercher les sites de production de transferrine dans le cerveau du rat adulte, en identifiant sur coupes histologiques, la transferrine par cytoimmunologie et l'ARNm correspondant par hybridation in situ. Les résultats obtenus établissent qu'une catégorie de cellules gliales responsable des phénomènes de myélinisation, les oligodendrocytes, synthétisent et stockent la transferrine.

\section{-Matériel et méthodes.}

Étude immunohistochimique : nous avons recherché la transferrine sur des coupes de cerveau de rat mâle adulte, par immunofluorescence indirecte ou technique immunoenzymatique, en utilisant 5 anticorps anti-transferrine distincts, dont un anticorps monoclonal, selon des modalités et avec les contrôles de spécificité appropriés décrits par ailleurs [9]. L'ensemble des expériences comportait le traitement de coupes de foie de rat à titre de contrôle.

Hybridation in situ : nous avons recherché la présence d'ARNm de transferrine sur des coupes de cerveau de rat, en utilisant comme sonde un ADN complémentaire (ADNc) de l'ARNm de la transferrine de rat marqué au phosphore 32 . L'ADNc marqué mis en contact avec les coupes dans des conditions d'incubation appropriées, s'hybride spécifiquement avec l'ARNm correspondant. Le signal est détecté par autoradiographie par noircissement d'un film rayon-X et par impression d'une émulsion liquide mise au contact de la coupe. L'ensemble de la procédure et des contrôles appropriés a été décrit par ailleurs $[9,10]$. 
Figure I. Détection de la transferrine dans les oligodendrocytes, par immunofluorescence indirecte.

Cortex pariétal (gauche) et chiasma optique (droite) - Cerveau du rat adulte.

Figure 2. Détection de l'ARNm de la transferrine par hybridation in situ avec un $A D N c$ de la transferrine de rat.

Les zones noircies $d u$ film rayon- $X$ correspondent aux zones marquées de la coupe. Coupe parasagittale de cerveau de rat adulte. Les zones les plus riches en $A R N m$ de la transferrine sont les faisceaux de substance blanche dont, ici, le corps calleux et la substance blanche du cervelet.

Figure 3. Coupe traitée par hybridation in situ avec l'ADNc de la transferrine:

Observation en fond clair après autoradiographie et coloration au bleu de toluidine. Substance blanche. On observe une importante accumulation de grains au niveau des oligodendrocytes, traduisant la présence de l'ARNm de la transferrine.

$m / s n^{0}$ \& juin-juillet 85
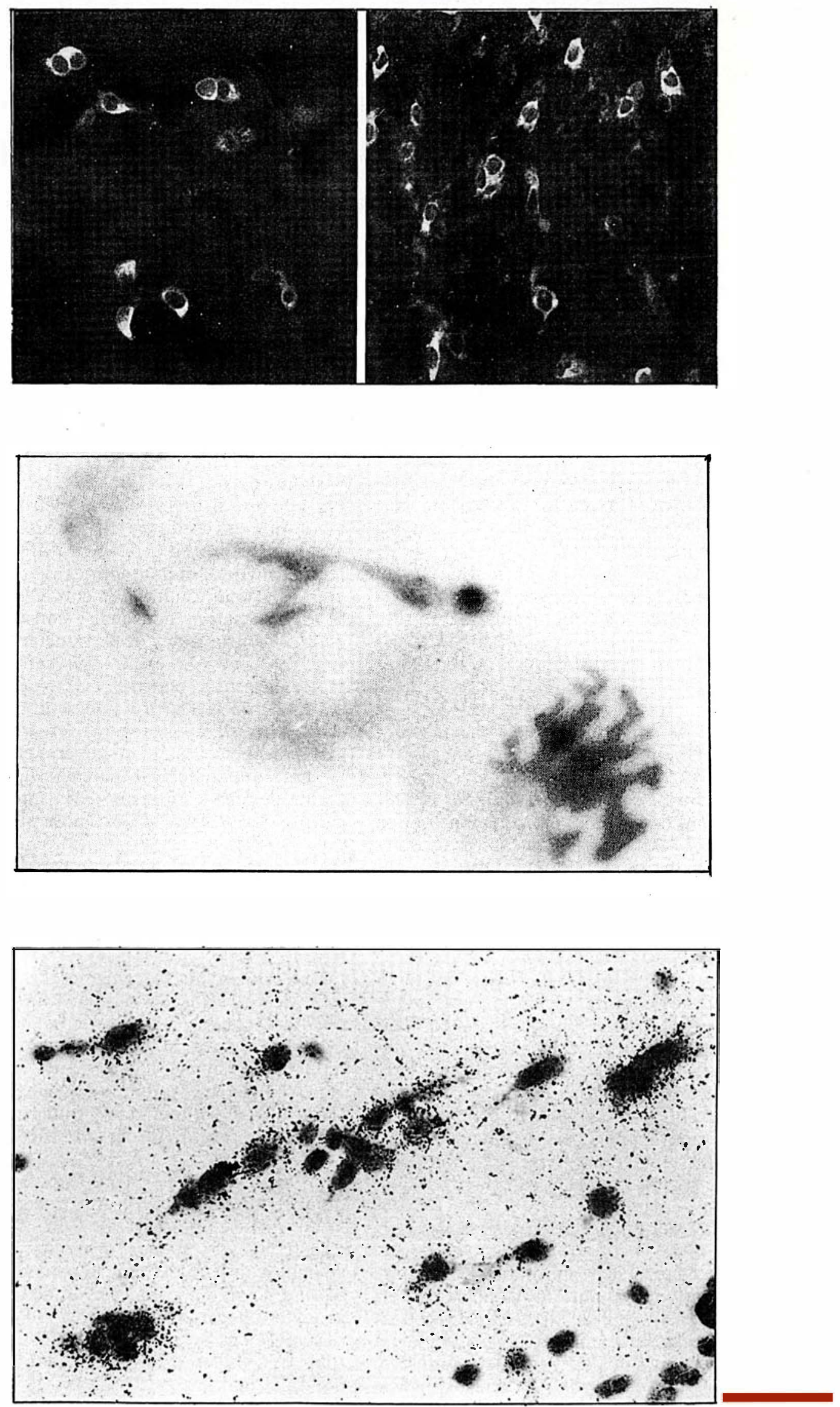


\section{Résultats}

Les divers anticorps antitransferrine utilisés marquent, au niveau du foie le cytoplasme des hépatocytes et dans le système nerveux, les plexus choroïdes ainsi que le cytoplasme et l'émergence des prolongements de cellules gliales ayant les caractéristiques des oligodendrocytes. Ces cellules sont présentes à la fois dans la substance blanche et la substance grise. Elles sont de petite taille, d'aspect arrondi ou stellaire, comportant des prolongements rares, fins et courts (figure 1 , voir p. précédente). Dans la substance grise, de telles cellules sont souvent situées au voisinage des capillaires. Dans la substance blanche, elles apparaissent souvent alignées par groupes, entre les faisceaux d'axones myélinisés. La myéline elle-même n'est pas marquée.

Les résultats de l'hybridation in situ pour la détection de l'ARNm de la transferrine montrent, sur film rayon-X, un marquage préférentiellement localisé dans les territoires de substance blanche tels le corps calleux, la capsule interne, le chiasma optique et la substance blanche du cervelet (figure 2, voir $p$. précédente). L'examen des préparations au microscope après autoradiographie montre une localisation de l'ARNm au niveau de cellules gliales dont l'organisation, la topographie et l'abondance sont identiques à celles des cellules marquées par les anticorps anti-transferrine (figure 3, voir p. précédente). Ceci est net en particulier dans des territoires comme le chiasma optique, le corps calleux ou la substance blanche du cervelet dans laquelle les oligodendrocytes sont particulièrement abondants. Les neurones, les cellules épendymaires et les cellules endothéliales des capillaires apparaissent dépourvus de marquage après hybridation in situ.

\section{Discussion}

La présence simultanée dans une même population cellulaire, de la transferrine et de l'ARNm codant pour cette molécule, témoigne de l'expression du gène de la transferrine et démontre qu'il existe, dans le cerveau du rat adulte, une synthèse endogène extrêmement active de cette molécule par les oligodendrocytes. Ces résultats montrent que l'expression du gène de la transferrine constitue un marqueur des oligodendrocytes. Une telle localisation élective suggère que la transferrine produite dans le cerveau pourrait avoir un rôle spécifiquement en relation avec le fonctionnement du système nerveux. Nos observations sont à rapprocher, d'une part de l'existence de récepteurs pour la transferrine dans certains neurones [11], et dans les cellules endothéliales des capillaires cérébraux [12] (à l'exclusion de tout autre territoire vasculaire), et d'autre part des propriétés différenciatrices de la transferrine au niveau du tissu musculaire [4].

Par ailleurs, il a été montré clairement que les cellules gliales sont capables de produire in vitro différents facteurs (non identifiés à ce jour) [13] conditionnant la survie et la différenciation neuronale; considérant les propriétés de la transferrine, il n'est pas exclu que cette molécule puisse constituer l'un de ces facteurs. Cette protéine semble essentielle à la croissance et au métabolisme cellulaire en général et, en tant que telle, est partie intégrante de tous les milieux définis de culture de cellules. Il est possible que la transferrine circulante synthétisée par le foie, traverse mal la barrière hémoméningée de telle sorte qu'une secrétion paracrine locale serait indispensable au maintien de la fonction des cellules cérébrales. Quant au mécanisme d'action de la transferrine, soit uniquement comme une molécule de transport du fer, soit aussi comme un véritable facteur de croissance, il reste controversé, quoique plusieurs éléments expérimentaux militent plutôt en faveur de la première hypothèse [14].

En conclusion, ce travail montre qu'il existe dans le cerveau des cellules gliales synthétisant de la transferrine de manière quantitativement comparable aux hépatocytes. La sécrétion paracrine de cette protéine pourrait jouer un rôle essentiel dans le maintien des fonctions de cellules cérébrales, notamment des neurones.

\section{REFERENCES}

1. Huebers HA, Finch CA. Transferrin: physiologic behavior and clinical implications. Blood 1984; 64:763-7.

2. Barnes D, Sato G. Serum-free cell culture: a unif ying approach. Cell 1980; 22: 649-55.

3. Ekblom P, Thesleff I, Saxen L, Miettinen A Timpl R. Transferrin as a fetal growth factor: acquisition of responsiveness related to embryonic induction. Proc Natl Acad Sci USA I 983;80:2651-5.

4. Beach RL, Popiela H, Festoof BW. The identification of neurotropic factor as a transferrin. FEBS Lett 1983; 156: 15 1-6.

5. Mac Knight GS, Lee DC, Palmiter RD. Transf errin gene expression. Regulation of mRNA transcription in chick liver by steroid hormones and iron deficiency. $7 \mathrm{Biol}$ Chem 1980; 225:1 48-53

6. Skinner MK, Griswold MD. Secretion of testicular transferrin by cultured Sertoli cells is regulated by hormones and retinoids. Biol Reprod 1982;27: 2 11-2 !

7. Levin MJ, Tuil D, Uzan G, Dreyfus JC, Kahn A. Expression of the transf errin gene during development in non-hepatic tissues: high level of transferrin mRNA in fetal muscle and adult brain. Biochem Biophys Res Commun 1984; 122 : 21 2-7.

8. Tuil $\mathrm{D}$, Vaulont $\mathrm{S}$, Levin $\mathrm{MJ}$, et al. In vivo control of rate transferrin gene expression: cyclic AMP, but not iron and steroïd hormones, regulates transcription of the gene. Embo 7 1985; sous presse.

9. Bloch B, Popovici T, Levin MJ, Tuil D, Kahn A. Production of transferrin by oligodendrocytes in the rat brain. Proc Natl Acad Sci USA 1985; sous presse.

10. Bloch B, Le Guellec D, De Keyzer Y. Detection of the messenger RNAs coding for the opioid peptide precursors in pituitary and adrenal by in situ hybridization: study in several mammal species. Neurosci Lett 1985; 53: 14 1-8.

I1 . Markelonis GJ, Oh TH, Park LP, et al. Synthesis of the transferrin receptor by cultures of embryonic chicken spinal neurons. 7 Cell Biol 1985 ; 100:8-17.

12. Jefferies WA, Brandon MR, Hunt SV, Williams AF, Gatter KC, Mason DY. Transferrin receptor on endothelium of brain capillaries. Nature 1 984; 312 : 162-3.

13. Arenander AT, De Vellis J. Glial-released proteins in neural intercellular communications: molecular mapping, modulation and influence on neuronal differentiation. In: Proteins in the nervous system: structure and function. New York: Alan R Liss Inc, 1982: 243-69.

14. Ozawa E, Kimura I, Hasegawa T, et al. Ironbound transferrin as a myotrophic factor. In: Ebashi S, Ozawa E, eds. Muscular Dystrophy, biomedical Aspects. Berlin: Springer Verlag et Tokyo: Japan Sci Soc Press, 1983: 53-60. 